\title{
THE USE OF OPTICAL FIBRES IN RADIOASTRONOMY
}

\author{
ALAN C. YOUNG \\ Australia Telescope National Facility ${ }^{*}$ \\ PO Box 76, Epping 2121, Australia
}

\begin{abstract}
Optical fibres have been used extensively within the Australia Telescope to carry IF data, LO reference, timing signals and computer communications. This paper discusses the various options considered in the design of a system which should assist similar projects in making the best use of optical fibres.
\end{abstract}

\section{INTRODUCTION}

The Australia Telescope (AT) is now operational and is the first radiotelescope to make extensive use of optical-fibre technology. It is to be expected that virtually all future, and most existing, radiotelescopes will use optical fibres in some form and will benefit from the AT's experience.

The AT optical-fibre project was faced with a large number of decisions. Due to changing technology and differing requirements, future projects will almost certainly make quite different decisions. With this in mind, this paper will concentrate on the choices that are available rather than on the actual system chosen for the AT.

It is widely known that optical fibre is used primarily because of three things: low cost, low signal-loss, and large bandwidth. For example, the cost of an optical-fibre cable capable of transferring 500 megabits per second over $2 \mathrm{~km}$ is probably no more than one fifth of the cost of coaxial cable of comparable capacity. Loss is typically $0.5 \mathrm{~dB}$ per kilometre, the potential bandwidth of single-mode optical fibre is extremely large, and it does not have to be equalized as does coaxial cable, with its steep increase of loss with frequency. Also, optical fibre is not only immune to electrical interference, but also produces no interference - added benefits in a radiotelescope environment.

However, any technology which is used in a new application has its share of problems. The poor signal-to-noise ratio and linearity partly explains the widespread use of simple digital modulation in most fibre applications. For a radiotelescope application optical-fibre connectors are also less than ideal.

\footnotetext{
* The Australia Telescope National Facility is operated in association with the Division of Radiophysics by CSIRO.
} 


\section{RADIOASTRONOMY USES}

The first question to consider is: what areas within an interferometer can benefit from the use of optical fibre?

For a connected array the most obvious use is for the return of IF signals from individual antennas to a central correlator or similar device. Optical fibre's low signal-loss means that long cable runs (up to typically $50 \mathrm{~km}$ ) are possible without repeaters, and the freedom from a heavy frequency-dependant loss removes the need for complex equalization systems. Bandwidths in excess of $10 \mathrm{GHz}$ are now quite possible and a bandwidth of around $1 \mathrm{GHz}$ is easily achieved with inexpensive, readily available components.

Another potential use is for the transfer of local oscillator reference frequency signals to all antennas within a round-trip, phase-stabilization system. The lower temperature coefficient and low signal-loss at frequencies around $1 \mathrm{GHz}$ make optical fibre an attractive medium for this application. Early AT development work revealed some difficult problems due to the combination of low signal-loss and high levels of reflection in optical fibre systems; these problems have only recently been overcome .

Components are now greatly improved and the phase-disturbing mechanisms better understood so that a transfer stability of the order of $1 \mathrm{pS} \mathrm{rms} \mathrm{(i.e.} 0.3^{\circ}$ per $\mathrm{GHz}$ ) is now quite achievable. Work at the Jet Propulsion Laboratory (Primas et al. 1989; see also Primas et al. 1990) suggests that stabilities an order of magnitude better are possible with fixed end stations and without connectors. Optical fibre with a very low temperature coefficient is now available and may remove the need for round-trip correction in short links (Lutes \& Primas 1989).

Lasers and optical receivers are not common to both round-trip paths and some care is needed to ensure good phase stability within these components. Care is also required to control the effect of reflections at connector interfaces.

Computer links, LANs, timing and other less demanding communication links are other obvious applications. The major advantages are low cost and good electromagnetic compatability.

\section{MODULATION CHOICES}

Simple digital amplitude modulation (where the optical carrier is switched on and off) is by far the most common modulation technique. This form of modulation has the advantage that the cable delay variations can be easily removed by combining clock recovery from the fibre and synchronizing bits. Digital modulation implies that the sampler is within the antenna and this can cause some problems due to interference, possible loss of bandwidth flexibility and greater difficulty in synchronisation between antennas. 
A coding scheme such as 'Manchester' or ' $5 \mathrm{~B} / 6 \mathrm{~B}$ ' coding is necessary to ensure reliable clock recovery at the receiver, but these are well developed in the communications industry. More complex digital modulation schemes such as phase switching, digital FM and quadrature amplitude modulation (QAM) are also possible but are rarely used because of the combination of wide bandwidth and low signal-to-noise ratio of optical fibre.

Analog amplitude modulation suffers from poor signal-to-noise ratio and often from poor linearity. Frequency modulation of a subcarrier is often used to reduce these effects, but at the expense of much reduced bandwidth. Newer components have greatly reduced the reflections which cause fine-structure passband-ripples when using amplitude modulation, but reflections at connectors can still cause problems. A delay-correction scheme is also usually necessary for analog systems, to correct for cable drift. Analog modulation is normally necessary for LO reference transfer.

\section{SINGLE MODE OR MULTIMODE ?}

Single-mode fibre with a diameter of $125 \mu \mathrm{m}$ and a light carrying core of $10 \mu \mathrm{m}$ or less is the most widely used type of fibre for wide bandwidth transmission. Terahertz bandwidths are ultimately possible but transmit and receive devices limit the bandwidth currently available to around $10 \mathrm{GHz}$ or lower. The only significant disadvantage of single-mode fibre is that the usual butt connectors for this fibre are quite fragile; this is discussed later.

Systems using multimode fibre where the core diameter is $50 \mu \mathrm{m}$ or larger can avoid this problem by using robust expanded-beam (lensed) connectors as described below. The major disadvantage of multimode fibre is that it is inherently bandwidth-limited due to modal dispersion. However, a bandwidth of $1 \mathrm{GHz}$ over a distance of $2 \mathrm{~km}$ (or $100 \mathrm{MHz}$ over $20 \mathrm{~km}$ ) is now readily achievable. Modal noise (Epworth 1981) can also seriously impair multimode systems.

As with normal copper cables, optical-fibre cables usually contain many fibres protected in slots or tubes within a heavy protective jacket. It is quite possible to have more than 30 fibres in a cable of $10 \mathrm{~mm}$ diameter.

\section{CONNECTORS}

Using optical fibre in a radiotelescope with movable antennas presents a problem, particularly where the movement occurs regularly. The ability to disconnect the antenna is usually required. Most connectors for optical fibre need great care if they are to last for more than a few matings. This care involves a dust free environment and careful cleaning of the connector at every mating. Alternatives to connectors such as trailing cables are cumbersome.

The majority of fibre connectors achieve a simple butt connection of two highly polished fibre ends using high-precision connector inserts. A 
loss of less than $0.1 \mathrm{~dB}$ is typical for a mated pair. Both fibre end faces and alignment inserts are easily damaged by dust and abrasion. This is not a significant problem for the typical telecom fixed link but, in an application requiring frequent disconnection, connector lifetime can be as little as 10 to 50 matings unless adequate care is taken. Recent connector designs may have improved this situation but it is an area where testing should be carried out if frequent disconnection is likely.

Expanded-beam connectors use lenses to achieve greater robustness by expanding the light-carrying beam, exiting from the fibre core, to typically $0.5 \mathrm{~mm}$ in diameter. The light beam then travels across a small air space at the connector interface before being refocused into the second fibre core. A moderate amount of dust is no longer a problem and a light wipe is all that is needed for cleaning. Condensation can be a problem but the AT has not yet experienced any such failure even in very wet conditions. These connectors have a typical loss of $1 \mathrm{~dB}$ per mated pair and designers of analog systems should also be wary of significant reflections in expanded-beam connectors.

Until recently, robust expanded-beam connectors were only available for multimode fibre. However, a four-way, military-style, single-mode, expanded-beam connector has recently been released in the UK by Stratos.

Fibre connections which do not need to be disconnected can either be butt-connected with one of the mechanical splices available, or fusionspliced. Fusion splicing is a well-developed technique but it does require access to a good fusion-splicing machine and these are still expensive. Splice losses are generally much less than $0.1 \mathrm{~dB}$.

Careful interpretation of connector loss is necessary, especially in multimode fibre. Measured losses are dependant on modal distribution and freedom from cladding modes in the test fibres (Cooper 1983), and are not necessarily the losses of the connectors when installed in the system. The measured loss of the total system may be considerably more than the sum of the nominal component losses. It may even be possible to add a connector and receive more signal to imply a gain rather than a loss.

\section{LIGHT SOURCES AND RECEIVERS}

The light-emitting diode (LED) is the simplest light source, it is inherently eye safe and, since it is an incoherent source, it does not produce modal noise in multimode fibre. Unfortunately, the output power is typically only $10 \mu \mathrm{W}$ and the maximum modulation rate is around $100 \mathrm{MHz}$.

Semiconductor laser diodes are normally used for the higher speed systems. Output power is usually in the 0.5 to $5 \mathrm{~mW}$ range and bandwidths extend to beyond $10 \mathrm{GHz}$, but devices in the $1 \mathrm{GHz}$ range are more common. Such lasers do not have sufficient power to damage normal tissue, but it is possible to damage sensitive eye tissue and it is wise to consider some protection procedures such as labelling and automatic shutdown circuitry. Most laser diode devices contain Peltier cooling and internal sensors for temperature and output power, although cooling may no longer be as necessary as it was for earlier, low-efficiency devices. 
Most lasers are still of the simpler 'Fabri-Perot' construction but distributed feedback (DFB) lasers, which have extremely narrow spectral width, are becoming more commonplace, especially for long distance links. However, there is a danger that the improved coherence of these devices may cause them to be more easily disturbed by reflections, especially in short link applications.

The receiving element is either an avalanche photodiode (APD) or PIN diode and these are followed by low-noise, high-gain amplifiers. These amplifiers can be either conventional $50 \Omega$ microwave amplifiers or special purpose transimpedance or FET input amplifiers with equalization. Minimum usable input optical power depends on the actual devices used but is typically $-45 \mathrm{dBm}$ for a bandwidth of $10 \mathrm{MHz}$ and reduces to around $-30 \mathrm{dBm}$ at $1 \mathrm{GHz}$ bandwidth for a system with $20 \mathrm{~dB}$ signal-to-noise ratio.

\section{WAVELENGTH}

Lasers and LEDs are unly available at a few wavelengths. The least expensive components are at $0.85 \mu \mathrm{m}$ and most computer links are at this wavelength. Most telecom and other wide bandwidth links have now moved to the $1.3 \mu \mathrm{m}$ wavelength where the chromatic dispersion of the fibre is a minimum. This wavelength also has much lower fibre loss and is the usual choice for long, wideband links. At present, the range of specialised components is also best at this wavelength. For the longest links, the $1.55 \mu \mathrm{m}$ wavelength has the lowest loss, although dispersion can limit the bandwidth unless narrow spectral-width lasers are used. Components are expensive at this wavelength and the range of components is limited.

\section{MULTIPLEXING}

Most radiotelescope systems will require more than one communication path, and some form of multiplexing onto a single or small number of fibres should be considered. Multiplexing can reduce the number of fibres and hence the number of connectors.

If the signals are all digital, then time-division multiplexing is the obvious first candidate. This is not difficult at low-bit rates but is more challenging at gigabit rates unless a suitable commercial multiplexer can be found. Time multiplexing also requires a much wider bandwidth fibre link.

Wavelength-division multiplexing is the equivalent of frequencydivision multiplexing on a microwave carrier. Several options are possible. Many IF channels could be carried on different optical bearers separated in wavelength by, say, $10 \mathrm{~nm}$ in the $1.3 \mu \mathrm{m}$ band. This technique is expected to be widely used in future since it has immense bandwidth capability, but the components needed are only slowly becoming available. Alternatively, one IF channel could use the $1.3 \mu \mathrm{m}$ band with another IF channel on a carrier in the $1.55 \mu \mathrm{m}$ band.. A third 
alternative is to multiplex lower-bit rate $(<100 \mathrm{Mbit} / \mathrm{S})$ computer links, and so on, on the same fibre at a wavelength of $850 \mathrm{~nm}$ where components are less expensive.

Signals at different wavelengths can flow in opposite directions if necessary, provided that the wavelength-separating demultiplexers are bidirectional. In fact, it should be possible to use the same wavelength in opposite directions on the same fibre using available high-directivity couplers and power splitters. However, this will present problems unless reflections are kept very low.

\section{CABLING STRATEGY}

For a radiotelescope with movable antennas, there are many more antenna stations than antennas and a cabling strategy must be chosen. The choices are essentially the same as for coaxial cable except that, whereas fibre is relatively inexpensive, connectors are more lossy and expensive, and so the balance is often tipped towards a system which uses more fibre and fewer connectors.

The simplest cabling strategy is to install a separate cable from each station, with the cables from the many unused stations lying idle. This uses a large amount of fibre and requires some sort of switchboard at the centre to connect to the cables which run to the active stations. An alternative is to have a 'trunk' cable and use links at each unused station. This will require many robust connectors in series. The loss is likely to be around $1 \mathrm{~dB}$ per station and this may be unacceptable if there are many stations. The use of couplers at each station is another alternative but the loss is higher and so, usually, is the cost.

\section{THE AUSTRALIA TELESCOPE SYSTEM}

The system chosen for the $3 \mathrm{~km}$ AT subarray uses one four-fibre cable from each station. Each fibre conveys a single, digitized IF channel to the central site at a wavelength of $1.3 \mu \mathrm{m}$. Manchester coding was chosen since, although it is wasteful of bandwidth, it is easier to implement at these high rates than alternative codes. Manchester coding converts the 512 Mbit per second sampler output rate to a 1024 Mbit per second rate on the fibres.

Wavelength multiplexing is used to carry bidirectional computer links and timing signals at a wavelength of $0.85 \mu \mathrm{m}$ on the same fibres. Wide bandwidth multimode fibre avoids the connector problems of single mode fibre. The total link loss due to the $2 \mathrm{~km}$ cable length, connectors, multiplexers and splices is around $10 \mathrm{~dB}$ at $1.3 \mu \mathrm{m}$ and $15 \mathrm{~dB}$ at $0.85 \mu \mathrm{m}$.

Each station post uses a four-way, expanded-beam, military-style connector, having $1 \mathrm{~dB}($ at $1.3 \mu \mathrm{m})$ typical loss. A patch panel exchange at the central building uses inexpensive, expanded-beam connectors. The graded-index multimode fibre has a $50 \mu \mathrm{m}$ core, $125 \mu \mathrm{m}$ glass cladding and an acrylate buffer. There is a total of $40 \mathrm{~km}$ of four-fibre cable which is $12 \mathrm{~mm}$ in diameter, uses 'kevlar' reinforcing and a rigid nylon jacket. 
Slotted core construction was used for the underground cable and tight buffer construction for the vertical runs. Tight buffer avoids excessive weight on the fibre ends.

Laser sources are used for the high-bit rate $1.3 \mu \mathrm{m}$ channels and LED sources at $0.85 \mu \mathrm{m} ; 1.3 \mu \mathrm{m}$ receivers use APD detectors and simple $50 \Omega$ amplifiers. The $1.3 \mu \mathrm{m}$ system has now been operating for two years with excellent reliability. Bench tests showed a link bandwidth of $1 \mathrm{GHz}$ and an error rate of 1 in $10^{15}$.

A fixed antenna, which is $4.5 \mathrm{~km}$ from the central site, is used to extend the baseline to $6 \mathrm{~km}$. Single-mode fibre is being used for this link because of the longer distance and the reduced connector problem. Multiplexing is not used for this link. A separate pair of fibres has also been installed for the distribution of LO reference to this site, as described below.

Optical fibre is also being used for many short links within antennas and buildings to reduce interference and cost. For example, many of the interconnections into the correlator-screened room are soon to be converted to optical fibre.

\section{LO TRANSFER}

Tests carried out for a round-trip system for LO reference transfer indicate that separate fibres for the forward and return paths do not degrade performance, provided that they are closely coupled thermally (Sarma 1988). In fact, performance of a single fibre test system was slightly worse, due to the inadequate isolation and higher reflections in the directional couplers necessary in such a system.

For the AT $6 \mathrm{~km}$ site, the LO transfer system will send a $160 \mathrm{MHz}$ reference signal along one fibre to the antenna. A narrow-bandwidth phase-lock loop at the antenna will recover the signal and produce a very low-phase noise signal for use within the LO systems. Some of this reference signal will be sent back along the second fibre to the central site and compared with the original signal in a precision phase meter. From any measured phase change, an appropriate correction will be calculated and applied to the antenna LO phase rotators. Present plans call for the installation of this round-trip system and antenna commissioning during 1991.

\section{FUTURE TRENDS}

Trying to predict the future is always difficult and, probably, the only certainty is that the use of optical fibre will continue to increase at a fast rate. However, it is possible to predict the changes that are most likely to occar.

The bandwidth of optical-fibre systems has rapidly increased so that $10 \mathrm{GHz}$ systems should become more commonplace. Direct increase much beyond this will be slower because of the cost and the difficulty of using mm-wave devices. However, other methods of obtaining greater 
use of the potential bandwidth, such as wavelength multiplexing, are likely to gain ground. For example, it may be possible, ultimately, to multiplex more than 100 channels, each of $10 \mathrm{GHz}$, on a single fibre. Coherent detection still appears to be a long way from large-scale commercial use but this would help to make multiplexing easier and improve the signal-to-noise ratio of analog systems.

The cost and availability of optical components should continue to improve but the cost of the optical fibre itself has probably "bottomed". Specialised fibre, such as the low temperature coefficient fibre recently released by Sumitomo, may soon become more readily available and hence less expensive. Optical switches, amplifiers, filters and test equipment are still either quite crude, expensive or unobtainable. The future should see a drive to improve these components, making the development of optical switching systems and, ultimately, optical signal processing and computing systems, possible.

\section{ACKNOWLEDGMENTS}

The author wishes to thank R. Ticehurst, N.V.G. Sarma, M. Anderson, M. Hayes and P. Miller for major contributions to this work. The work was supported entirely by the Australia Telescope National Facility.

\section{REFERENCES}

Cooper, R. 1983, Fiber Optic Insertion Loss Measurements: how they relate to system use, AMP Inc. (Harrisburg, PA) Technical Paper P 270-83, p. 20814.

Epworth, R.E. 1981, Laser Focus, 17, 109.

Lutes, G and Primas, L 1989, State-of-the-Art Fiber Optics for Short Distance Frequency Reference Distributuion, JPL Telecommunications and Data Acquisition Progress Report 42-97, Jan-Mar., p. 81.

Primas, L.E, Lutes, G.F, and Sydnor, R.L. 1989, Stabilized Fiber- Optic Frequency Distributuion System, JPL Telecommunications and Data Acquisition Progress Report 42-97, Jan-Mar., p. 88.

Primas, L.E, Logan, R.T, Lutes, G.F, and Maleki, L. 1990, in Proceedings, Fiber Optics Conference '90, McLean VA March 1990, p. 352.

Sarma, N.V.G. 1988, Optical Fibre Link For Reference Frequency Distribution, Australia Telescope Technical Report AT/23.2/020. 
Masato Ishiguro: Would you please comment about the roundtrip phase correction scheme in optical-fiber transmission system.

A. Young: The techniques are almost the same as for a coaxial based round trip system with lasers and detectors added. Problems stem from the very low loss of fibre and high reflections from some components. Our most recent experiments show that we can stabilize a $5 \mathrm{~km}$ link to better than $1 \mathrm{pS} \mathrm{rms}$ phase stability (for $<1$ hour time scales). It appears to be better to use a separate fibre for each direction due to the better isolation achievable and the lower reflection magnitude without couplers. 